\title{
Cancer prevention, aerobic capacity, and physical functioning in survivors related to physical activity: a recent review
}

This article was published in the following Dove Press journal:

Cancer Management and Research

5 June 2010

Number of times this article has been viewed

\author{
Matthew S Wiggins' \\ Emily M Simonavice ${ }^{2}$ \\ 'Department of Kinesiology, \\ University of Wisconsin-Eau Claire, \\ Eau Claire, WI, USA; ${ }^{2}$ Florida State \\ University, Tallahassee, FL, USA
}

Correspondence: Professor Matt Wiggins Department of Kinesiology, University of Wisconsin-Eau Claire, 105 Garfield Ave, PO Box 4004 Eau Claire,WI 54702, USA $\mathrm{Tel}+\mid$ 7I5-836-3I59

Fax + I 7|5-836-4074

Email wigginsm@uwec.edu

\begin{abstract}
According to recent published reports, over 12 million new cases of cancer were estimated worldwide for 2007. Estimates from 2008 predict that cancer will account for $22.8 \%$ of all deaths in the US. Another report stated 50\% to $75 \%$ of cancer deaths in the US are related to smoking, poor dietary choices, and physical inactivity. A 2004 report indicated obesity and/ or a sedentary lifestyle increases the risk of developing several types of cancer. Conversely, several large-scale cohort studies point to the positive relationship between physical activity and a reduction in cancer risk. In addition, research over the last few years has clearly shown cardiorespiratory benefits, increases in quality of life (QOL), and increases in physical functioning for cancer survivors who engage in exercise programs. Thus, the purpose of this review is to highlight three areas related to cancer and physical activity. First, information concerning the prevention of cancer through physical activity is addressed. Second, recent studies identifying changes in volume of oxygen uptake $\left(\mathrm{VO}_{2}\right)$ and/or cardiorespiratory functioning involving exercise with cancer survivors is presented. Third, studies identifying changes in cancer survivors' physical functional capacity and QOL are presented. Finally, a summary of the review is offered.
\end{abstract}

Keywords: cancer, cardiorespiratory, exercise, physical activity, volume of oxygen $\left(\mathrm{VO}_{2}\right)$

\section{Introduction}

According to the most recently published reports on the internet, over 12 million new cases of cancer were estimated worldwide for the year $2007 .{ }^{1}$ Incidence and mortality rates continue to grow around the globe, with lung cancer deaths estimated to be near 1 million per year. The American Cancer Society estimated that cancer would account for or be associated with $22.8 \%$ of all deaths in the US, just trailing cardiovascular disease as the number one killer which accounted for $26.6 \%$ of the total deaths. Between 2000-2004 death rates for cancer were the highest in the US for individuals of African American heritage, while people from the Asian and Pacific Island ethnic group had the lowest incident rates, which were approximately half of that for African Americans. According to 2008 estimates, cancers of the prostate and breast will continue be the most frequently diagnosed cancers in men and women accounting for approximately $25 \%$ in each group. ${ }^{2}$

While it appears that the prevalence of cancer is not in decline, various organizations and researchers are attempting to limit this disease through the improvement of healthy behavior choices. Although not all risk factors can be controlled or eliminated that predispose an individual to cancer (ie, genetic factors), healthy behaviors and lifestyle choices, along with preventing environmental exposure to certain risk factors, can help curtail cancer development in humans. Unfortunately, in many cases exposure 
to specific cancer causing agents is almost undetectable and therefore unavoidable. However, in other cases, individuals can be proactive regarding their health by choosing not to smoke, or by becoming engaged in an exercise program. These last two choices become even more important when one considers that $50 \%$ to $75 \%$ of cancer deaths in the US are related to risk factors such as smoking, poor dietary choices, and physical inactivity. ${ }^{3}$ Thus, not only do we see the reduction of risks and increase in health benefits in the area of heart disease through being physically active (eg, lowering cholesterol, less chance of stroke or heart attack), we also see positive results in the area of lowering obesity levels, which in turn decreases one's risk of cancer. ${ }^{4}$

In addition, research over the last few years has clearly shown cardiorespiratory benefits (increases in aerobic capacity), increases in quality of life (QOL), and increases in physical functioning for cancer survivors who engage in exercise programs. ${ }^{5-7}$ Furthermore, a 2004 report on diet and nutrition indicated that obesity and/or a sedentary lifestyle (both modifiable through diet and exercise) increases the risk for developing several types of cancer such as breast, colon, kidney, and endometrial cancer. ${ }^{4}$ Thus, evidence continues to accumulate through research studies and reviews indicating a link between a physically active healthy lifestyle and improvements in mental and physical QOL in cancer survivors. ${ }^{8-10}$ However, we should note that although some studies interchange the terms physical activity and exercise, there is a clear delineation. Physical activity is generally considered any type of body movement that requires energy expenditure. ${ }^{11}$ Examples tend be recreational (eg, walking, gardening), occupational, household (eg, mowing the lawn, doing chores), or lower intensity activities such as golf. Exercise, on the other hand, can be defined as movements designed to improve physical fitness measures such as body fat percentage, flexibility, muscular endurance, and aerobic capacity. ${ }^{11}$ Exercise is typically planned, structured, and repetitive, with prescribed levels of intensity, frequency and duration. Examples of exercise include, although are not limited to, structured workouts at a wellness facility (eg, weight training), power walking, cycling, cross-country skiing, or jogging. We shall use these definitions when referring to physical activity and exercise in this article.

The purpose of this review is to highlight three specific areas. First, information concerning the prevention of cancer through the avenue of physical activity will be addressed, mainly through previous reviews and cohort studies. Second, recent studies identifying changes in volume of oxygen uptake $\left(\mathrm{VO}_{2}\right)$ involving exercise with cancer survivors will be presented. Studies identifying changes in cancer survivors' aerobic capacity as measured by sub-maximal or field cardiorespiratory tests will also be presented in this section. Third, research associated with changes in physical functioning and/or QOL, especially those showing an increase relative to changes in aerobic capacity will be reviewed. A summary section is then presented to close out the discussion. This review is intended to present findings that can communicate a general consensus related to the area of exercise, physical activity, and cancer prevention or recovery. It is beyond the scope of this review to focus on genetic, metabolic or chemical factors that may predispose or increase the risk of developing cancer, ${ }^{12-14}$ nor will we identify all research findings connected with exercise or physical activity and cancer. For instance, some research focuses on the potential mechanisms by which physical activity may reduce cancer development. ${ }^{15-17}$ It is hoped, however, that a connection between cancer prevention and increases in aerobic capacity (ie, $\mathrm{VO}_{2}$ or cardiorespiratory functioning) can be postulated from this review.

\section{Cancer prevention research}

According to the Cancer Trends Progress Report - 2007 published by the National Cancer Institute (NCI), obesity and physical inactivity are associated with approximately $25 \%$ to $30 \%$ of colon, breast, endometrial, kidney, and esophageal cancers in the US. ${ }^{3}$ Other research indicates that higher levels of physical activity may lower the risk for developing prostate and lung cancer. ${ }^{18}$ In addition to the information presented by the NCI, recent literature reviews also provide supporting evidence for the proposition that physical activity helps to prevent cancer. ${ }^{9,19}$ The findings of epidemiologic studies consisting of large-scale cohorts, meta-analyses, reviews, and/or case studies summarize the factors associated with a decreased risk for the development of various types of cancer. In the next few paragraphs, information is presented regarding the specific association between physical activity levels and the occurrence of breast, colon, and rectal cancer.

Physical activity has been reported for several years now as a viable means to reduce the development of breast and colon cancer, ${ }^{9,17,20}$ and theoretically should also help lower the reoccurrence of cancer. ${ }^{21-23}$ It should be noted, however, that not all research unequivocally report the benefits of an active lifestyle in the prevention of breast cancer. For instance, Lahmann and colleagues initially found that total and recreational physical activity did not affect breast cancer risk in premenopausal women. ${ }^{24}$ Additionally, Margolis and associates 
reported that physical activity levels in individuals under 30 years of age were not associated with breast cancer risk. ${ }^{25}$ However, in both preceding studies, positive results related to physical activity levels were later identified through separate data analyses. Thus, very few studies report physical activity as having a non-significant role in the prevention of breast cancer as Lee and colleagues did in $2001 .{ }^{26}$ Lee reported 2 years later that approximately two thirds of the studies reviewed at that time showed a significant negative relationship between physical activity levels and the risk for developing breast cancer. $^{20}$

More recent reports have also provided strong evidence suggesting the link between physical activity levels and risk reduction through literature reviews of breast cancer research. ${ }^{9,19,21}$ Specifically, Friedenreich in 2004 reported that although several studies found significant general associations with exercise and breast cancer, the most profound reductions in breast cancer development due to increased physical activity levels were found in postmenopausal women. ${ }^{21}$ In her review of recent cancer prevention literature, Kruk provides several examples of global cohort studies reporting breast cancer risk reduction through a physically active lifestyle, recreational activities, or higher levels of household activities. ${ }^{9}$ Miles concludes her review by stating that the majority of research presented strongly supports the notion that physical activity helps prevent breast cancer. ${ }^{19}$ According to Miles and other researchers, there also appears to be an inverse dose-response relationship between physical activity and breast cancer. ${ }^{19,24,27,28}$ Therefore, higher levels of physical activity intensity, frequency, and duration (ie, exercise at moderate to vigorous levels) appear to be more beneficial to modifying one's risk for developing breast cancer than being involved in lower levels of recreational activity.

As stated previously, research generally supports the notion that higher physical activity levels are negatively related to colon cancer risk. For instance, Lee reported that as physical activity increases in both men and women, the risk of colon cancer is reduced by as much as $80 \%$, with a median reduction in most studies equating to a $30 \%$ to $40 \%$ reduction..$^{20} \mathrm{~A}$ study by Calton and associates somewhat refutes this statement, as they found no relationship between colon cancer and physical activity in a large cohort of US women. ${ }^{29}$ More recently, however, Kruk reported in her review of chronic diseases that evidence for the reduction of colon cancer through a physically active lifestyle is convincing, with an average risk reduction of $40 \%$ to $50 \%{ }^{9}$ Miles verifies this finding in her literature review of several cohort studies, whereby being physically active reduces one's colon cancer risk by approximately $40 \% .{ }^{19}$ Also of importance is her note distinguishing that these investigations consisted of cohorts from the US, Asia, and Europe. Thus, generalizability to culturally divergent populations can be considered valid, while also indicating the findings to be robust.

Most research reports are unclear concerning the doseresponse needed to lower overall colon cancer risk; however, general recommendations taken from reviews seem to indicate higher intensity levels over a prolonged period of time offer greater cancer reduction effects. ${ }^{19}$ For instance, Samad and associates identified both occupational and recreation physical activity variables as leading to lower colon cancer risk for men and women when combining results from 19 cohort studies. ${ }^{16}$ Similar to the findings reported on breast cancer risk reduction, Lee and colleagues found a significant negative relationship between more strenuous exercise or daily physical work and the risk of developing colon cancer in a cohort of Japanese men. ${ }^{30}$ In addition, physical activity has also been shown to improve QOL within this cancer population. Reporting on colon and rectal cancer together, Lynch and associates found a positive relationship between improvements in QOL and physical activity from a cohort of subjects in Australia. ${ }^{31}$ Specifically, participants achieving a minimum of 150 minutes of physical activity per week improved QOL scores by approximately $20 \%$.

Although sometimes associated or grouped with colon cancer, statistics for rectal cancer are not as promising. In fact, several reports show no consistent significant association between physical activity and the prevention of rectal cancer. ${ }^{9,30,32}$ In one meta-analysis by Samad and colleagues, no relationship was found in case-control or cohort studies between physical activity and rectal cancer for either men or women. ${ }^{16}$ In 2004, Wei and associates combined two largescale cohorts in their analysis of colon and rectal cancer..$^{33}$ Although results indicated a significant reduction in colon cancer, physical activity levels were not associated with rectal cancer risk. In the same year, Chao and colleagues reported that physical activity was significantly related to a decrease in rectal cancer, but that colon cancer was not related. ${ }^{34}$ Other studies such as that by Lund Nilsen and Vatten show similar mixed findings related to colorectal cancer. ${ }^{35}$ Thus based on the existing data, there is no strong evidence to suggest a definitive link between increased physical activity levels and a reduced risk of rectal cancer.

In addition to the aforementioned cancers (breast, colon, and rectal), physical activity has been associated with reducing the risk of lung, prostate, and endometrial cancer. The average risk reduction for each of these cancers varies 
from approximately $10 \%$ to $40 \%$. An initial cohort study by Colbert and colleagues in 2003 were unable to show a doseresponse relationship concerning total or vigorous physical activity and endometrial cancer. ${ }^{36}$ More recently, Miles indicated that a majority of research for endometrial cancer supports the risk-reducing effects of physical activity. ${ }^{19}$ However, it was unclear whether occupational or recreational activity provided the greatest benefits, or whether increases in risk were more related to a sedentary lifestyle. As for prostate cancer, separate reports by Lee along with Torti and Matheson indicated a decreased risk associated with physical activity in a majority of the studies they reviewed. ${ }^{20,23}$ Nilsen and associates found an inverse relationship with a Norwegian cohort, and also reported that there was a significant decrease in advanced prostate cancer occurrence with higher levels of physical activity. ${ }^{37}$ Although there are a few studies indicating an inverse relationship (ie, lower cancer risk associated with higher levels of activity), several recent studies found no association between physical activity and the prevention of prostate cancer. ${ }^{38-40}$ According to Friedenreich and Orenstein, the physical activity relationship with prostate and endometrial cancers was only considered probable or possible. ${ }^{18}$

A review by Lee has suggested a slightly stronger association between physical activity and lung cancer, however, it also indicates that smoking was not controlled for in several studies. ${ }^{20}$ More recently, Miles reported that the reduced risk of lung cancer may more heavily depend on the intensity of the activity or exercise session, as opposed to the total physical activity levels reported. ${ }^{19}$ Specifically, levels of strenuous or vigorous activity (ie, exercises like cycling or various recreational sports) are more strongly related to lung cancer risk reduction than general activities such as those related to one's occupation, work around the house, or leisure pursuits. ${ }^{41,42}$ Therefore, physical activity sessions which specifically require a lung cancer survivor to exercise at a given intensity, frequency, and duration may be most beneficial for producing not only cardiorespiratory benefits for the heart and lungs (ie, increased stroke volume, aerobic capacity), but also for producing physiological benefits to help reduce the risk of developing cancer.

\section{Aerobic capacity/VO 2 research}

Originally, one of the intentions of this review was to determine if there was sufficient evidence to state that an increase in aerobic capacity could help lower one's risk of developing cancer. Unfortunately there is not sufficient evidence to make that statement. However, as presented in the preceding paragraphs, higher levels of physical activity help to prevent many cancers. It stands to reason that increases or higher levels of aerobic capacity, attained through sufficient amounts of physical activity or exercise, should therefore help to prevent cancer. Moreover, increases in physical activity should also provide higher levels of aerobic and physical functioning, and increase QOL for cancer survivors. Thus, this section of the review is dedicated to presenting research findings related to increases in aerobic capacity of cancer survivors, either through maximal volume of oxygen measures $\left(\mathrm{VO}_{2}\right)$, or through sub-maximal and field cardiorespiratory tests. While exercise and physical activity may not be as strongly related to some cancers, any reduction in the risk of developing cancer should be deemed important, especially given the other benefits related to exercise (ie, reduced incidence of heart disease, diabetes, obesity).

There are several positive physiological changes for many cancer survivors associated with moderate to high levels of physical activity and structured exercise. One of these physiological changes can be an increase in cardiorespiratory functioning, or an increase in aerobic capacity. Simply stated, exercise has the capacity to positively impact one's heart and lung ability to deliver blood and oxygen to the body. Increases in aerobic capacity for cancer survivors, normally measured through $\mathrm{VO}_{2}$ analyses, have been shown in several studies. For example, Young-McCaughan and associates in 2003 found a significant increase in $\mathrm{VO}_{2}$ for a group of 62 cancer survivors, with some going through treatment, after a 12 -week exercise program. ${ }^{43}$ The authors also reported an overall increase in physical functioning for the participants representing survivors of several different types of cancer. A few years later, Thorsen and colleagues reported a significant increase in $\mathrm{VO}_{2}$ for 111 survivors in both the intervention and control groups in a home-based exercise program for breast, testicular, lymphoma, and gynecologic cancer. ${ }^{7}$ In this study, all survivors had recently completed their chemotherapy treatment. The average increase in $\mathrm{VO}_{2}$ was 6.4 milliliters $(\mathrm{mL})$ per kilogram $(\mathrm{kg})$ of body weight for the intervention group who participated in a 14-week training program, and $3.1 \mathrm{~mL} / \mathrm{kg}$ for the control group who were told to maintain their normal physical activities.

More recently, Cheema and Gaul indicated that 25 previously trained breast cancer subjects significantly increased their $\mathrm{VO}_{2}$ measures by an average of $6 \%$ from pre- to post-test after an 8 -week exercise program. ${ }^{44}$ Subjects participated in 2 resistance or weight training sessions and 3 aerobic sessions per week. Herrero and associates found 
aerobic capacity measures (expressed in both ventilatory and respiratory compensation thresholds) significantly related to QOL scores in 16 untrained breast cancer participants. ${ }^{45}$ The authors also reported $\mathrm{VO}_{2}$ peak values (ie, maximal testing scores) related to QOL as approaching a significant level. In a recently published health education study, Garner and Erck found that 11 Stage I and II breast cancer survivors significantly increased their aerobic capacity $\left(\mathrm{VO}_{2}\right)$ by an average of $4 \mathrm{~mL} / \mathrm{kg}$ through a moderate to vigorously intense 8 -week aerobic and resistance training program. ${ }^{46}$ Wiggins and Simonavice, using a sub-maximal formula, recorded an average $\mathrm{VO}_{2}$ increase of $5 \mathrm{~mL} / \mathrm{kg}$ in 6 breast cancer survivors after 3 months of a 12-month exercise program containing both an aerobic and resistance exercise component. ${ }^{47}$ The resulting increase in cardiorespiratory capacity was generally maintained by the participants throughout the next 9 months of the program.

In addition to the previous investigations comparing $\mathrm{VO}_{2}$ differences, several studies indicating changes in cancer survivors' aerobic capacity, as measured by other cardiorespiratory indices, have been published. For example, Pinto and colleagues showed improvements in blood pressure levels with 24 breast cancer survivors after a 3-month exercise program, which is suggestive of cardiorespiratory improvements in the subjects. ${ }^{48}$ Patients involved in another 3 -month exercise program that gradually increased intensity and duration throughout the study significantly increased their aerobic capacity. ${ }^{49}$ In the same year, Courneya and associates found a non-significant increase in treadmill time with 93 colorectal cancer survivors who exercised moderately at home 3 to 5 times per week for 16 weeks. ${ }^{5}$ A more recent case study by de Paleville and associates in 2007 showed an overall increase of $40.9 \%$ in 12 -minute walking time with a breast cancer patient. ${ }^{50}$ This change occurred over nine weeks with increases in duration of aerobic training (ie, walking), and while the patient was going through chemotherapy treatment. The reviewed literature provides encouraging results indicating that survivors of various types of cancer, as well as individuals still going through treatment, have the potential to increase their aerobic capacity by engaging in structured exercise programs. In addition to these studies cited, a meta-analysis by Tardon and colleagues reported that increased pulmonary function following high levels of physical activity (ie, exercise) could result in a decreased opportunity for airway exposure to inhaled carcinogens. ${ }^{51}$ One could therefore conjecture that increases in cardiorespiratory capacity should help to lower one's risk for developing cancer.

\section{Physical functioning/QOL research}

A fair amount of research with cancer survivors has investigated the effects of exercise on what is generally termed physical functioning or capacity. In some cases this refers to some type of cardiorespiratory measure, while in other studies it may be defined as functioning related to muscular strength and endurance. For instance, in a group of 31 breast cancer survivors, Schwartz reported an increase in functional capacity (12-minute walk) in an exercise adoption group, and a decreased functional capacity in those not adhering to exercise. ${ }^{52}$ In the same year, Schwartz also found a decrease in percentage body fat and body weight control in 71 breast cancer patients completing an 8-week home-based exercise program..$^{53}$ She reiterated the significance of these findings in her article, noting that excess body fat produces estrogen and may increase the risk of breast cancer. Mock and associates demonstrated increased physical functioning with 50 breast cancer participants using a home-based exercise program. In their study, the low intensity walking group reported a decreased activity level (ie, lower physical functioning) while the high intensity walking group increased their activity level and 12-minute walk test scores. ${ }^{54}$ Exercise tolerance for intensity and duration increased significantly for 62 survivors in the investigation by Young-McCaughan and colleagues. ${ }^{43}$ A qualitative study by Adamsen and associates with 23 survivors of varying cancers showed increases in physical capacity and increased energy levels after a 6-week exercise program that also included relaxation and massage therapy. ${ }^{55}$ In addition to these physiological investigations, Courneya and his group of investigators reported that exercise has even been shown to increase "perceived" physical functioning in cancer survivors. $^{5}$

Besides changes in physical functioning, several articles mention muscular strength and endurance increases as a result of adopting an exercise program. For instance, Cheema and Gaul reported significant improvements in upper and lower body muscular endurance with 27 previously trained breast cancer survivors after an 8 -week exercise program. ${ }^{44}$ The authors also noted that a few participants had lymphedema to start the study, although they showed no increase in arm circumference after the study. Kirshbaum reported that exercise should generally help patients with lymphedema, while Lane and associates indicated that the remaining lymphatic vessels may weaken if not physically trained, thus increasing the likelihood of developing lymphedema (see also the position statement on lymphedema and exercise by the NLN Medical Advisory Committee).${ }^{56-58}$ A study from Denmark by Quist and colleagues reported a slight increase 
in aerobic fitness for 79 patients undergoing chemotherapy. ${ }^{6}$ More importantly, subjects participating in the nine hours per week training program that lasted 6 weeks also showed an average increase in muscular strength of $41 \%$. Wiggins and Simonavice found upper body muscular strength and lower body endurance increases in 6 breast cancer survivors from baseline to 12 months in a structured exercise program. ${ }^{47}$ Most recently, Johnson and associates reported that moderate to vigorously intense activity was significantly associated with improvements in physical functioning in older, longterm colorectal cancer survivors. ${ }^{59}$

In addition to the physical functioning and muscular improvements reported, several studies indicate exercise can also produce favorable results concerning fatigue levels, psycho-social measures, and QOL. More specifically, fatigue is the most frequently reported symptom of cancer treatment. ${ }^{60}$ In many cases rest is the most common medical advice given for the treatment of fatigue, ${ }^{8,61-63}$ which can lead to de-conditioning and a lower functional capacity. ${ }^{64}$ Interesting to note, however, is that fatigue can be characterized as a positive perception when associated with exercise. ${ }^{55}$ According to Douglas, care should be taken when prescribing exercise duration. ${ }^{8}$ She indicates that higher levels of exercise ( $>60$ minutes) increases fatigue and decreases QOL. Schwartz reported less QOL decline in patients improving their functional capacity, ${ }^{65}$ while Young-McCaughan and colleagues indicated that higher QOL can be achieved with increased exercise tolerance levels. ${ }^{43}$ More recently, Wiggins and Simonavice have shown in two studies that QOL in cancer survivors can be increased and maintained from several months to a year via a structured exercise program. ${ }^{10,47}$ In terms of psychosocial measures, improvements have been reported for cancer survivors' related anxiety and depression levels, ${ }^{5,54,62}$ body image or body esteem, ${ }^{48}$ emotional and social well-being, ${ }^{5}$ and self-efficacy related to exercise barriers as a result of exercise engagement. ${ }^{66}$ From the literature reviewed in the preceding paragraphs, it appears that physical activity and exercise seem to have a promising role in the improvement of physical function, psychological well-being, and overall QOL in cancer patients and survivors.

\section{Summary}

As presented in this review, select research over the last 10 years clearly provides strong evidence for physical activity helping to lower the risk of breast and colon cancer. In addition, the literature indicates that being physically active can also reduce one's risk of lung, prostate, and endometrial cancer, with rectal cancer research showing some favorable although mixed results. Several articles report a dose-response intervention whereby greater levels of moderate to vigorous activity helps in furthering the reduction of cancer risk. The implications from these findings seem fairly straight-forward. In order to help lower the chances of developing cancer in one's lifetime, the adoption of a healthy and physically active lifestyle is encouraged. Whether through recreational pursuits, structured exercise programs, or through health education programs, activity and information needs to be disseminated to the general public regarding the benefits of being physically active and improving one's aerobic fitness levels. If possible, exercise intensity, frequency, and duration should be monitored to promote a moderate to vigorous level of activity. Higher levels of activity should further decrease one's cancer risk, as well as provide other cardiorespiratory and cardiovascular benefits.

Research also demonstrates that being physically active and participating in an exercise program can benefit cancer survivors. While exercise and cancer recovery programs specifically designed for survivors are few, a majority of the training sessions share similar characteristics. In general, activities have a combination of aerobic and muscular endurance exercises that most "apparently healthy" populations would use in a structured program. One very important difference for cancer survivors, when compared to an apparently healthy population, involves the prescribed intensity level used to monitor exercise sessions. Specifically, intensity values should be set at a lower threshold or range (ie, $40 \%$ to $60 \%$ instead of $60 \%$ to $80 \%$ ) to accommodate the typical treatment-related fatigue and de-conditioning that afflicts the cancer recovery participant. Intensity, frequency, and duration are then increased with time to improve the participants conditioning level. ${ }^{10,67}$ Hopefully the information that has been gathered over the past 2 decades will prompt a call for more proactive measures to provide survivors all the resources they need to combat this disease. As more information becomes available, and more practitioners become involved in cancer recovery and fitness programs, it is hoped that more survivors will be alerted to these types of programs through their oncologists and cancer support staff.

Although the studies presented in this review are not exhaustive, they do show the many benefits for survivors who participate in exercise programs, whether it be increases in aerobic capacity, QOL, and/or physical functioning. In fact, some of the most commonly reported side-effects associated with cancer treatment (eg, fatigue, lymphedema, peripheral neuropathy, anxiety and depression, sleep disorders, and body image problems) can be alleviated through exercise. 
Thus, cancer survivors can experience several of the benefits related to being physically active before, during, and after cancer and its treatment. Perhaps research in the next few years will be able to take exercise and cancer recovery findings one step further in this process. As demonstrated through this review, there appears to be a connection with increases in $\mathrm{VO}_{2}$ related to increases in physical functioning and QOL. ${ }^{43,44,47}$ As research in this area progresses, it is hoped that soon investigations will be able to demonstrate that higher cardiorespiratory functioning can be associated with decreases in cancer risk.

\section{Disclosures}

The authors disclose no conflicts of interest.

\section{References}

1. American Cancer Society. Global Facts and Figures 2007;2009. Available from http://www.cancer.org/downloads/STT/Global_Facts_and_ Figures_2007_rev2.pdf Accessed Dec 7, 2009.

2. American Cancer Society. Cancer Statistics 2008;2009. Available from http://www.cancer.org/docroot/PRO/content/PRO_1_1_Cancer_ Statistics_2008_Presentation.asp Accessed Dec 7, 2009.

3. National Cancer Institute. Cancer Trends Progress Report - 2007; 2009 Available from http://progressreport.cancer.gov/doc.asp?pid=1\&did=2 007\&mid=vcol\&chid=71 Accessed Dec 7, 2009.

4. Key TJ, Schatzkin A, Willett W, et al. Diet, nutrition and the prevention of cancer. Public Health Nutr. 2004;7:187-200.

5. Courneya KS, Friedenreich CM, Quinney HA, Fields ALA, Jones LW, Fairey AS. A randomized trial of exercise and quality of life in colorectal cancer survivors. Eur J Cancer Care. 2003;12:347-357.

6. Quist M, Rorth M, Zacho M, et al. High-intensity resistance and cardiovascular training improve physical capacity in cancer patients undergoing chemotherapy. Scand J Med Sci Sports. 2006;16:349-357.

7. Thorsen L, Skovlund E, Stromme SB, Hornslien K, Dahl AA, Fossa SD. Effectiveness of physical activity on cardiorespiratory fitness and health-related quality of life in young and middle-aged cancer patients after chemotherapy. J Clin Oncol. 2005;23:2378-2388.

8. Douglas E. Exercise in cancer patients. Phys Ther Rev. 2005;10:71-88.

9. Kruk J. Physical activity in the prevention of the most frequent chronic diseases: An analysis of the recent evidence. Asian Pacific Journal of Cancer Prevention. 2007;8:325-338.

10. Wiggins MS, Simonavice EM. Quality of life benefits in cancer survivorship with supervised exercise. Psychol Rep. 2009;104:421-424.

11. Fahey TD, Insel PM, Roth WT. Fit well: Core Concepts and Labs in Physical Fitness and Wellness. 8th ed. Boston: McGraw-Hill; 2009.

12. Campeau PM, Foulkes WD, Tischkowitz MD. Hereditary breast cancer: New genetic developments, new therapeutic avenues. Hum Genet. 2008; $124: 31-42$.

13. Kanetsky PA, Mitra N, Vardhanabhuti S, et al. Common variation in KITLG and at 5q31.3 predisposes to testicular germ cell cancer. Nat Genet. 2009;41:811-815.

14. Halliwell B. Oxidative stress and cancer: have we moved forward? Biochem J. 2007;401:1-11.

15. Rogers CJ, Colbert LH, Greiner JW, Perkins SN, Hursting SD. Physical activity and cancer prevention: Pathways and targets for intervention. Sports Med. 2008;38:271-296.

16. Samad AKA, Taylor RS, Marshall T, et al. A meta-analysis of the association of physical activity with reduced risk of colorectal cancer. Colorectal Dis. 2005;7:204-213.
17. Westerlind KC. Physical activity and cancer prevention-Mechanisms. Med Sci Sports Exerc. 2003;35:1834-1840.

18. Friedenreich CM, Orenstein MR. Physical activity and cancer prevention: Etiologic evidence and biological mechanisms. J Nutr. 2002;132:5456-5464.

19. Miles L. Physical activity and the prevention of cancer: A review of recent findings. Nutrition Bulletin. 2007;32:250-282.

20. Lee I-M. Physical activity and cancer prevention data from epidemiologic studies. Med Sci Sports Exerc. 2003;35:1823-1827.

21. Friedenreich CM. Physical activity and breast cancer risk: The effect of menopausal status. Exerc and Sport Sci Rev. 2004;32:180-184.

22. McTiernan A. Intervention studies in exercise and cancer prevention. Med Sci in Sports Exerc. 2003;35:1841-1845.

23. Torti DC, Matheson GO. Exercise and prostate cancer. Sports Med. 2004;34:363-369.

24. Lahmann PH, Friedenreich C, Schuit AJ, et al. Physical activity and breast cancer risk: The European prospective investigation into cancer and nutrition. Cancer Epidemiol Biomarkers Prev. 2007; 16:OF1-OF7.

25. Margolis KL, Mucci L, Braaten T. et al. Physical activity in different periods of life and the risk of breast cancer: The Norwegian-Swedish women's lifestyle and health cohort study. Cancer Epidemiol Biomarkers Preven. 2005;14:27-32.

26. Lee IM, Cook NR, Rexrode KM, Buring JE. Lifetime physical activity and risk of breast cancer. Br J Cancer. 2001;85: 962-965.

27. Chang SC, Ziegler RG, Dunn B, et al. Association of energy intake and energy balance with postmenopausal breast cancer in the prostate, lung, colorectal, and ovarian cancer screening trial. Cancer Epidemiol Biomarkers Prev. 2006;15:334-341.

28. McTiernan A, Kooperberg C, White E, et al. Recreational physical activity and the risk of breast cancer in postmenopausal women. JAMA. 2003;290:1331-1336.

29. Calton BA, Lacey JV Jr, Schatzkin A, et al. Physical activity and the risk of colon cancer among women: A prospective cohort study (United States). Int J Cancer. 2006;119:385-391.

30. Lee KJ, Inoue M, Otani T, et al. Physical activity and risk of colorectal cancer in Japanese men and women: the Japan Public Health Center-based prospective study. Cancer Causes Control. 2007;18: 199-209.

31. Lynch BM, Cerin E, Owen N, Hawkes AL, Aitken JF. Prospective relationships of physical activity with quality of life among colorectal cancer survivors. J Clin Oncol. 2008;26:4480-4487.

32. Friedenreich C, Norat T, Steindorf K, et al. Physical activity and risk of colon and rectal cancers: The European Prospective Internal Investigation into Cancer and Nutrition. Cancer Epidemiol Biomarkers Prev. 2006;15:2398-2407.

33. Wei EK, Giovannucci E, Wu K, et al. Comparison of risk factors for colon and rectal cancer. Int J Cancer. 2004;108:433-442.

34. Chao A, Connell CJ, Jacobs EJ, et al. Amount, type, and timing of recreational physical activity in relation to colon and rectal cancer in older adults: The Cancer Prevention Study II nutrition cohort. Cancer Epidemiol Biomarkers Prev. 2004;13:2187-2195.

35. Lund Nilsen TI, Vatten LJ. Colorectal cancer associated with BMI, physical activity, diabetes, and blood glucose. IARC Scie Publ. 2002;156:257-258.

36. Colbert LH, Lacey JV Jr, Schairer C, Albert P, Schatzkin A, Albanes D. Physical activity and risk of endometrial cancer in a prospective cohort study (United States). Cancer Causes Control. 2003;14:559-567.

37. Nilsen TIL, Romundstad PR, Vitten LJ. Recreational physical activity and risk of prostate cancer: A prospective population-based study in Norway (the HUNT study). Int J Cancer. 2006;119:2943-2947.

38. Littman AJ, Kristal AR, White E. Recreational physical activity and prostate cancer risk (United States). Cancer Causes Control. 2006; 17:831-841.

39. Patel AV, Rodriguez C, Jacobs EJ, et al. Recreational physical activity and risk of prostate cancer in a large cohort of US men. Cancer Epidemiol Biomarkers Prev. 2005;14:275-279. 
40. Zeegers MPA, Dirx MJM, van den Brandt PA. Physical activity and the risk of prostate cancer in The Netherlands Cohort Study, results after 9.3 years of follow-up. Cancer Epidemiol Biomarkers Prev. 2005;14:1490-1495.

41. Bak H, Christensen J, Lykke Thomsen B. et al. Physical activity and risk for lung cancer in a Danish cohort. Int $J$ Cancer. 2005;116:439-445.

42. Steindorf K, Friedenreich C, Linseisen J, et al. Physical activity and lung cancer risk in the European Prospective Investigation into Cancer and Nutrition cohort. Int J Cancer. 2006;119: 2389-2397.

43. Young-McCaughan S, Mays MZ, Arzola SM, et al. Change in exercise tolerance, activity and sleep patterns, and quality of life in patients with cancer participating in a structured exercise program. Oncol Nurs Forum. 2003;30:441-452.

44. Cheema BSB, Gaul CA. Full-body exercise training improves fitness and quality of life in survivors of breast cancer. $J$ Strength Cond Res. 2006;20:14-21.

45. Herrero F, Balmer J, San Juan AF, et al. Is cardiorespiratory fitness related to quality of life in survivors of breast cancer? J Strength Cond Res. 2006;20:535-540.

46. Garner D, Erck EG. Effects of aerobic exercise and resistance training on Stage I and II breast cancer survivors: A pilot study. Am J Health Educ. 2008;39:200-205.

47. Wiggins MS, Simonavice EM. Quality of life benefits: A 12 month exercise cancer recovery case study. KAHPERD Journal. 2008;44: 16-19.

48. Pinto B, Clark M, Maruyama N, Feder F. Psychological and fitness changes associated with exercise participation among women with breast cancer. Psychooncology. 2003;12:118-126.

49. Hayes SC, Rowbottom D, Davies PSW, Parker TW, Bashford J. Immunological changes after cancer treatment and participation in an exercise program. Med Sci Sports Exerc. 2003;35:2-9.

50. de Paleville DT, Topp RV, Swank AM. Effects of aerobic training prior to and during chemotherapy in a breast cancer patient: A case study. J Strength Cond Res. 2007;21:635-637.

51. Tardon A, Lee WJ, Delgado-Rodriguez M, et al. Leisure-time physical activity and lung cancer: A meta-analysis. Cancer Causes Control. 2005;16:389-397.

52. Schwartz AL. Daily fatigue patterns and effect of exercise in women with breast cancer. Cancer Pract. 2000;8(1):16-24.
53. Schwartz AL. Exercise and weight gain in breast cancer patients receiving chemotherapy. Cancer Pract. 2000;8:231-237.

54. Mock V, Pickett M, Ropka ME, et al. Fatigue and quality of life outcomes of exercise during cancer treatment. Cancer Pract. 2001;9: 119-127.

55. Adamsen L, Medtgaard J, Roerth M, Andersen D, Wuist M, Moeller T. Transforming the nature of fatigue through exercise: Qualitative findings from a multidimensional exercise programme in cancer patients undergoing chemotherapy. J Cancer Care. 2004;13:362-370.

56. Kirshbaum M. Promoting physical exercise in breast cancer care. Nursing Standard. 2005:19:41-48.

57. Lane K, Worsley D, McKenzie D. Exercise and the lymphatic system implications for breast-cancer survivors. Sports Med. 2005;35: 461-471.

58. NLN Medical Advisory Committee. Position statement of the National Lymphedema Network. Topic: Exercise; 2005. Available from: http:// www.lymphnet.org/pdfDocs/nlnexercise.pdf Accessed Oct 7, 2005.

59. Johnson BL, Trentham-Dietz A, Koltyn KF, Colbert LH. Physical activity and function in older, long-term colorectal cancer survivors. Cancer Causes Control. 2009;20:775-784.

60. Schneider CM, Dennehy CA, Carter SD. Exercise and Cancer Recovery. Champaign, IL: Human Kinetics; 2003.

61. Coon SK, Coleman EA. Keep moving: patients with myeloma talk about exercise and fatigue. Oncol Nurs Forum. 2004;31:1127-1135.

62. Courneya KS, Keats MR, Turner AR. Physical exercise and quality of life in caner patients following a high dose chemotherapy and autologous bone marrow transplantation. Psychooncology. 2000;9:127-136.

63. Watson T, Mock V. Exercise as intervention for cancer related fatigue. Phys Ther. 2004;84:736-743.

64. Courneya KS, Vallance JKH, Jones LW, Reiman T. Correlates of exercise intentions in non-Hodgkin's lymphoma survivors: An application of the theory of Planned Behavior. J Sport Exerc Psychol. 2005;27:335-349.

65. Schwartz AL. Fatigue mediates the effects of exercise on quality of life. Qual Life Res. 1999;8:529-538.

66. Wiggins MS, Rader E, Erdmann JB. Physical and psychological benefits in an exercise and cancer recovery program: Preliminary findings. Paper presented at the Association of Applied Sport Psychology National Conference, Louisville, KY, 2007; Oct 24-27.

67. Sprod LK. Considerations for training cancer survivors. J Strength Cond Res. 2009;31:39-47.
Cancer Management and Research

\section{Publish your work in this journal}

Cancer Management and Research is an international, peer-reviewed open access journal focusing on cancer research and the optimal use of preventative and integrated treatment interventions to achieve improved outcomes, enhanced survival and quality of life for the cancer patient. The journal welcomes original research, clinical \& epidemiological

\section{Dovepress}

studies, reviews \& evaluations, guidelines, expert opinion \& commentary, case reports \& extended reports. The manuscript management system is completely online and includes a very quick and fair peerreview system, which is all easy to use. Visit http://www.dovepress.com/ testimonials.php to read real quotes from published authors. 